\title{
Opioid and Drug Prevalence in Top 40's Music: A 30 Year Review
}

\author{
Curtis Hanba, MD and Daniel Hanba, MD
}

Objectives/Hypothesis: Our objective was to identify current trends in the prose of popular music and, specifically, to identify if a relationship exists between the mention of opioid narcotics in Top 40's music and a rising prevalence of opioid use disorder in the United States.

Methods: A list of Billboard's top 100 songs for each year of the past 30 years (1986 to 2016) was collected. Lyrics from the $\mathbf{4 0}$ most popular songs of each year were queried for reference to drugs and alcohol. $\chi^{2}$ analysis was used to identify statistically significant relationships with a level of significance set at $P<.05$.

Results: There was a statistically significant increase in the lyrical mention to opioids $(0.0 \%$ to $5.0 \%$ of songs, $P=.004)$, marijuana $(0.6 \%$ to $17.2 \%$ of songs, $P<.001)$, and alcohol $(3.1 \%$ to $23.6 \%$ of songs, $P<$ .001 ) in the 2010 s decade when compared with the songs analyzed in the 1980s. The mention of opioid drugs and medications emerged in the late 1990s, and since, $57.1 \%$ of opioid-referencing songs mention prescription opioid medications and not heroin or street slang reference of the drug. Male- and femaledriven mentions to drugs and alcohol have approached near equal rates in recent years.

Conclusion: There is increasing prevalence in the lyrical mention of opioids, among other drugs, in Top 40's music. Nearly $50 \%$ of 2016 Top 40's songs reference drugs or alcohol. Further inquiry may be warranted to evaluate the societal impact and persuasive abilities of popular culture, including Top 40's music, on American drug and alcohol use. (J Am Board Fam Med 2018;31:761-767.)

Keywords: Alcohol, Lyrics, Marijuana, Music, Opioid, Tobacco, Top 40s

The United States is in the midst of a widely publicized epidemic of opioid use disorder and overdose that is responsible for thousands of premature deaths and billions of dollars in economic losses annually. ${ }^{1,2}$ According to the Centers for Disease Control and Prevention (CDC), more than half a million people died from opioid overdose between the years 2000 and 2015, and the problem is getting worse, with an astonishing 33,091 people succumbing to opioids in 2015 alone. ${ }^{3}$ However,

This article was externally peer reviewed.

Submitted 1 January 2018; revised 21 April 2018; accepted 14 May 2018.

From Department of Otolaryngology-Head and Neck Surgery, The University of Minnesota Medical School, Minneapolis, MN (CH); Department of Internal Medicine and Pediatrics, Wayne State University School of Medicine, Detroit, MI (DH).

Funding: none.

Conflict of interest: none declared.

Corresponding author: Curtis Hanba, MD, Department of Otolaryngology, University of Minnesota, Phillips Wangensteen Building, 516 Delaware Street SE, Suite 8A, Minneapolis, MN 55455 (E-mail: cjhanba@umn.edu). although the ongoing opioid crisis continues to frequent newspaper headlines and is an enormous problem, it is worth noting that alcohol and tobacco remain more deadly and costly to Americans than opioids at this time. According to the National Institute of Alcohol Abuse and Alcoholism, approximately 88,000 people die yearly in the United States from alcohol-related causes, and, in 2010, alcohol misuse cost the United States \$249 billion. ${ }^{4}$ Nevertheless, tobacco still reigns king as the leading cause of preventable death in the United States, killing approximately 480,000 people and costing well over $\$ 300$ billion dollars annually.,

A fresh appreciation of these statistics underscores a need for physicians and health care officials to continue to unearth social and medical factors responsible for the significant mortality and financial burden related to these substances and their use. These evaluations, however, initiate a social "buzz" that in itself sparks an interesting debate among scholars: whether culture or the arts can impose a transformative message to a society. ${ }^{7}$ If so, 
what sort of buzz surrounds the current American opioid use disorder epidemic, and where can physicians and policy makers intervene?

Our objective was to identify current trends in the prose of popular music, with an emphasis on reference to opioids and drug use to examine a possible association between American drug use and Top 40's music. Our hope is that the relationships identified can facilitate an open discussion between physicians, patients, and policymakers on the current trends of American drug use to provide key tools to encourage safe and educated drug prevention strategies.

\section{Methods}

The Top 40 songs of the year as listed by Billboard for years 1986 to 2016 were identified and compiled into a Microsoft Excel (Seattle, WA) spreadsheet by author C.H. In the Billboard collection, songs are categorized as "Top songs" of the year based on national airplay, internet download, and purchase data. Song characteristics including artist sex, title, and year were manually entered into the spreadsheet during data collection by author C.H. Lyrics were collected from a variety of publicly available websites and evaluated for spelling during data entry. To attribute artist's sex, the primary vocalist was reviewed and documented for each song. No attempt was made to identify lyrical authors. Lyrical characteristics including the mention of opioids, alcohol, or tobacco were identified by author C.H. by using spreadsheet query formulas with strict inclusion criteria as detailed in Appendix A. Many of the queried terms were reviewed on a song by song basis for contextually. For example, terms like "pot," “medicine," “dope," “drink," "smoke," and many other terms that may not have been in reference to drugs or alcohol were evaluated within lyrical context to ensure appropriate attribution. Multidrug terms like "dope" were evaluated in the context of each song to accurately portray the referenced drug.

Longitudinal assessments were conducted by grouping songs into decade and yearly cohorts. The 1980s decade consisted of years 1986 to 1989 , whereas the 2010s decade incorporated years 2010 to 2016. For these 2 "decades," smaller sample sizes were assessed (140 and 240 songs, respectively) when compared with the 1990s and 2000s full collection of 400 songs. Songs were categorized into groups whether or not the lyrics contained a reference to each drug of interest individually. Songs with multiple references to a single drug of interest were tallied in a similar manor as songs containing one reference to each drug.

\section{Statistical analysis}

Microsoft Word and SPSS version 23 (IBM SPSS Statistics for Macintosh, version 22.0, Amarok, NY) were used for statistical calculation. $\chi^{2}$ tests were used to analyze categorical variables with a level of statistical significance set at $P<.05$.

\section{Results}

Figure 1 details climbing rates of lyrical mention to drugs of interest and alcohol in recent years. With the exception of tobacco, all drugs had statistically higher rates of mention in the 2010s decade than in the years assessed in the 1980s. Alcohol mentions climbed from $3.1 \%$ to $23.6 \%(P<.001)$, marijuana references from $0.6 \%$ to $17.2 \%(P<.001)$, and opioids $0.0 \%$ to $5.0 \%(P=.004)$. Tobacco's prevalence had no statistical deviation from the previous 1980 s benchmark: $1.3 \%$ to $1.1 \%(P=.865)$. References to alcohol and its consumption increased most dramatically from the 1990s to the 2000 s $(6.0 \%$ to $17.8 \%$ of songs). This transition also witnessed the greatest increase in marijuana references $(6.5 \%$ to $14.0 \%$ of songs) in year-end Top 40 hits. However, opioid references, although only first noticed in late 1990s and early 2000s music, have increased most dramatically in recent years, leaping from $1.5 \%$ of year-end Top 40 hits in the 2000 s to $5.0 \%$ of year-end Top 40 hits in the evolving 2010s decade. Of note, $57.1 \%$ of songs detailing opioid mentions referenced prescription opioid medications and not heroin or street slang reference of the drug. In 2016, 19 of the Top 40 $(47.5 \%)$ songs of the year mentioned either opioids, marijuana, alcohol, or tobacco at least once in the prose of the song.

\section{Sex Analysis}

Regarding lyrical references by sex, Figure 2 demonstrates a primarily male-driven increase in drugof-interest-related references, with recent popularity among female acts as well (2000 to 2010). The decade of the 2000s harbored the greatest growth in alcohol and marijuana references for males, with female acts playing "catch up" in recent (2000 to 
Figure 1. Percentage of Top 40s songs with drug of interest mentions by year.
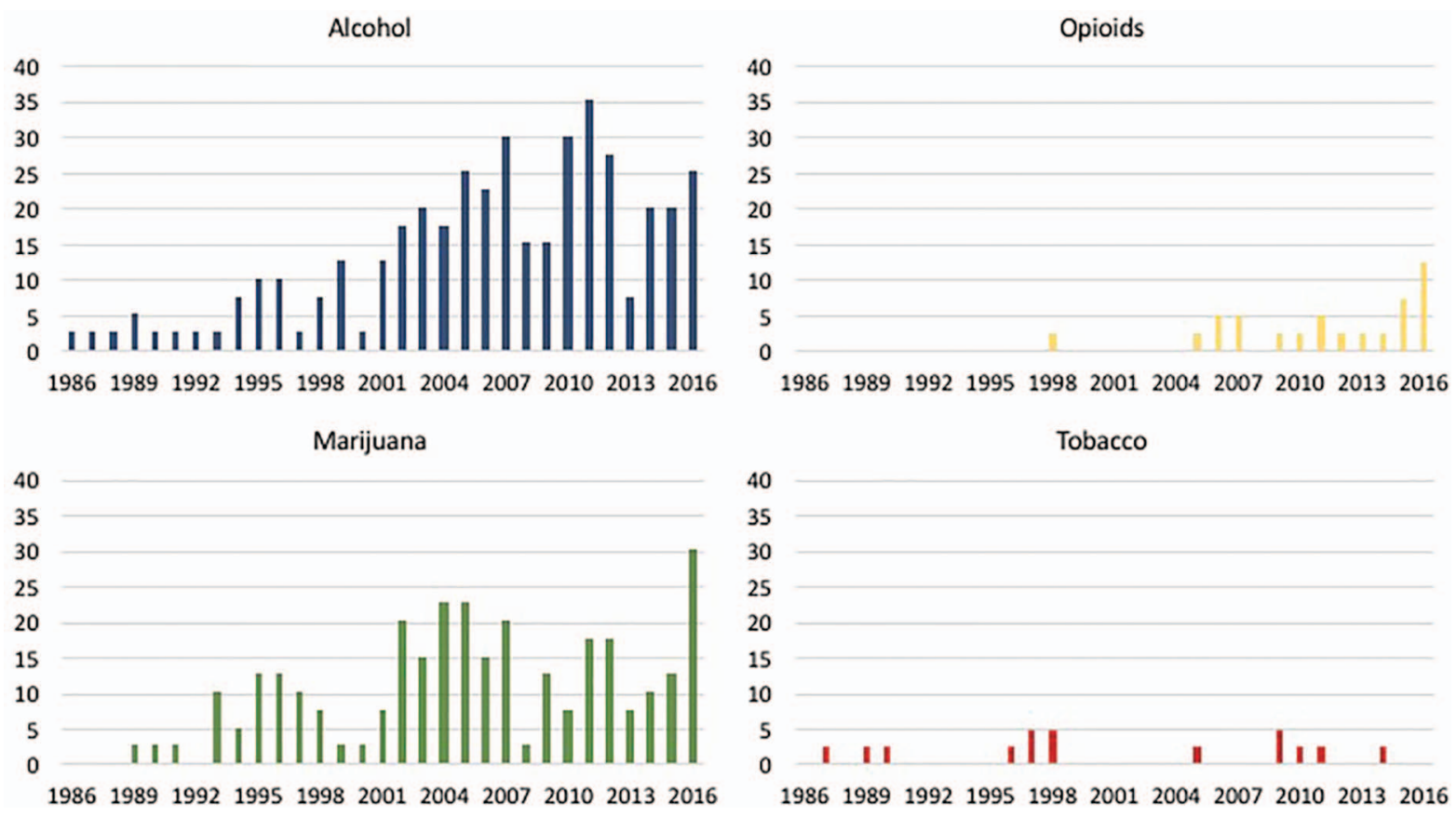

2010) years to near their male counterparts in mention percentages. Opioid mentions demonstrate a nearly exponential increase since the 1990s for males and have broken into female-driven lyrics within the last decade.

\section{Discussion}

Opioid reference has become increasingly common in today's Top 40's music. This trending upsurge roughly parallels an increased prevalence of opiate use disorder and overdose-related death in the United States. ${ }^{8}$ Although heroin is not a novel drug and this epidemic is not the first of its kind, recent opioid use has a new flavor, and its delivery has made it onto national airways. Over $50 \%$ of current Top 40's hit references discuss narcotic prescription or synthetic medication use, with the most common references including codeine, Percocet, and even remifentanil, among others. These powerful analgesics (with variable properties including half-life, delivery method, and potency) have a definite place in modern medicine; however, certain properties of these medications make them exceptionally dangerous to a vulnerable population of addicted and/or first-time users. In 2013, 92 persons suffered fatal overdoses related to fentanyl or fentanyl-like synthetics in the state of Ohio alone.

The year 2014 surfaced 514 fentanyl-related fatalities, nearly a $460 \%$ increase in fentanyl-related deaths from the year prior. ${ }^{9}$ Similarly, the Maryland Department of Health reported an increase in fentanyl-related overdose deaths from 58 in 2013 to 185 in 2014 , a $220 \%$ increase. ${ }^{10}$ As a result of the above, and with recognition of similar spikes in fentanyl-related overdose fatalities in other states, in early 2015, the CDC issued a Nationwide Alert labeling fentanyl as "a threat to public health and safety." 11 In addition, in the fall of 2015, the CDC issued a Health Advisory Statement to increase the awareness of fentanyl and synthetic opioid overdose occurrence, to improve the detection of fentanyl-related overdose outbreaks, and to augment naloxone training and administration practice among a variety of first responder and health care providers. ${ }^{12}$ Finally, in addition to increasing fentanyl and synthetic opioid overdose awareness, in 2016, the CDC launched a Clinical Practice Guideline to update and encourage proper narcotic prescription practice. Goals of these guidelines were to delineate when to initiate or continue opioids for chronic pain, discuss opioid selection, dosage, duration, follow-up, and discontinuation, and assess the potential risks and harms of chronic opioid use. ${ }^{13}$ The recommendations were to encour- 
Figure 2. Percentage of Top 40s songs with drugs of interest mentions by artist sex.
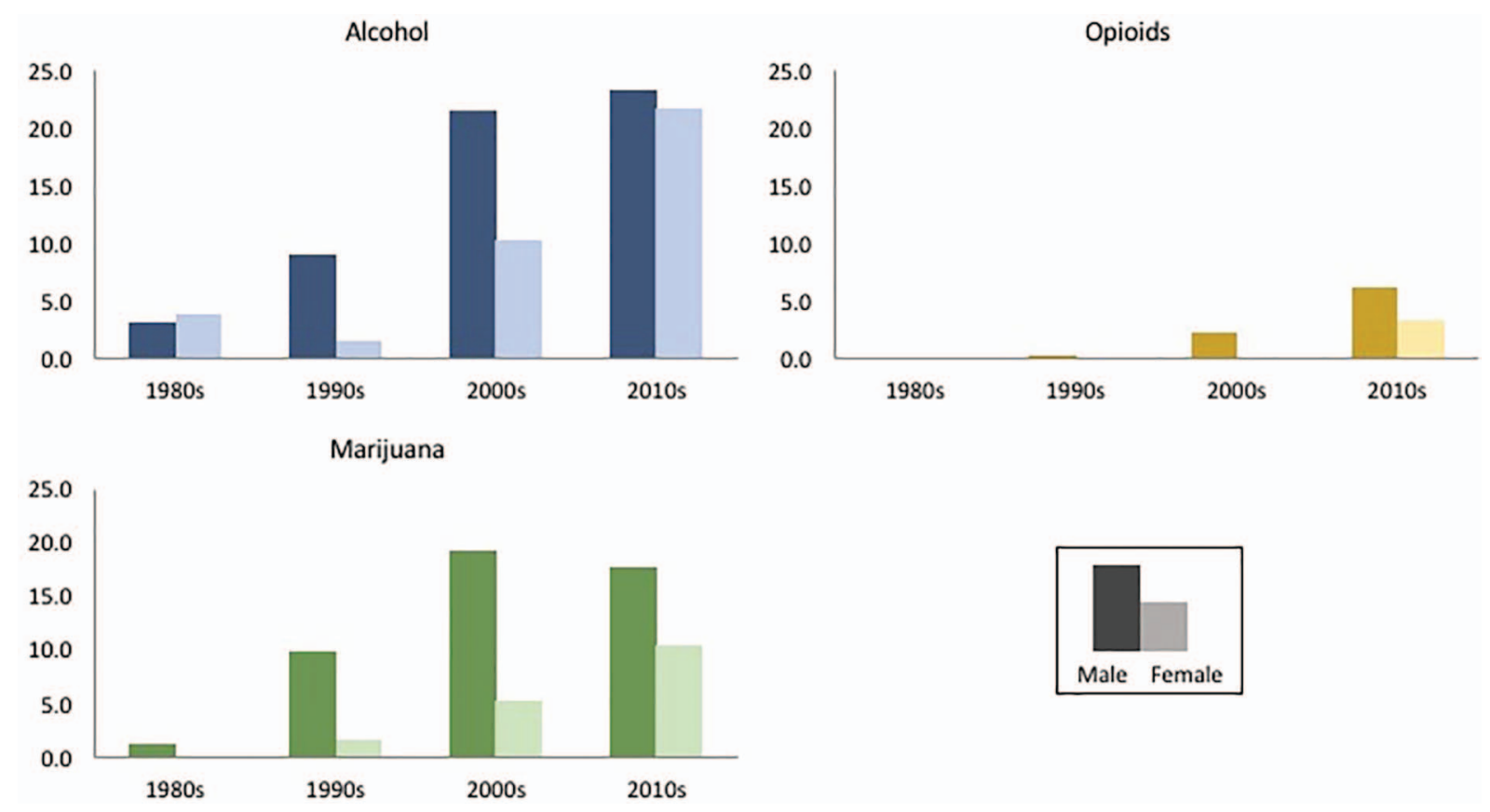

age low dosage, limited quantity, and goal-directed use of opioid medications. The guidelines also encourage alternative therapies to supplement opioid and nonopioid medications, thereby implementing a multidirectional attack on chronic pain and its causes. However, if reference to opioid medications continues to become "casual or trendy," as exemplified in the prose of many popularly aired lyrics, a more targeted public awareness campaign may be warranted to combat this growing national public health concern.

Public perception of recreational and medicinal marijuana use has evolved significantly during the time period during which we have seen increased lyrical mention of drugs in popular music. In 1969, Gallup polled Americans regarding marijuana legalization; at the time, $12 \%$ of the general public were in favor of legalizing the drug. ${ }^{14}$ By October 2016, Gallup polls showed $60 \%$ of Americans were in favor of legal recreational marijuana, with more than half of United States laws demonstrating some form of legalized marijuana. ${ }^{14}$ Similarly, the Pew Research Center identified that the adolescent American perception of the drug changed in recent years. In 1991, 84\% of adolescents identified the drug to be harmful to one's health, and this number dropped to $53.8 \%$ of adolescents in $2014 .{ }^{15}$ As demonstrated, public perception regarding mari-

juana has changed, from largely considering the drug a nuisance to society to the majority of Americans accepting the use either medically or recreationally, and this shift in public attitude may have influenced the acceptability of mentioning marijuana in Top 40's music, as displayed by our analysis. If this demonstrated "acceptability" trend is mirrored through a similar rise in the mention of opioid narcotics in Top 40's music, America's epidemic of overdose fatality may continue to escalate.

Although marijuana use has become increasing acceptable among American consumers, tobacco has become increasingly unpopular and stigmatized. In 1964, the Surgeon General of the United States released the landmark report Smoking and Health that described the findings of over 7000 scientific articles related to the adverse effects of smoking. ${ }^{16}$ Since the release of that monumental public health statement, the prevalence of smoking in the United States among adults has fallen from $43 \%$ to near $18 \%$ in $2014 .{ }^{17}$ Smoking habits demonstrate a much more common prevalence among those of lower socioeconomic status and lower education level ${ }^{18}$; however, strong data exists that shows smoking has been transformed in the public eye from a glamorous activity to one with a significant stigma attached. ${ }^{19-22}$ A declining prevalence of smoking in America is likely much attributable 
to the release of the historic public health statement Smoking and Health ${ }^{16}$. Its impact should serve as a beacon to policymakers and physicians to encourage continued release of scientifically backed public health statements. In addition, although these statements should ideally be universal, targeted efforts to reach underrepresented and poorly educated populations may be warranted to hopefully mitigate repetition of the shortcomings of Smoking and Health ${ }^{16}$.

The prevalence of opiate misuse disorder has skyrocketed, and we have seen a paralleled increase in the mention of opiates in popular music lyrics; however, this trend has not been reproduced when examining the mentions of alcohol over the same time frame. The National Survey on Drug Use and Health's data demonstrate a relatively steady percentage of alcohol-consuming Americans during the period of 2002 to 2014, with slightly downtrending use among those aged 12 to $17 .^{23}$ Concurrently, the frequency of alcohol mentions in popular music lyrics has increased substantially. One potential explanation to support these findings could be an influential negatively depicted portrayal of popular music lyrics regarding one's choice to drink alcohol or consume drugs. This possibility may have contributed to a stagnant percentage of drinking Americans, while simultaneously influencing America's underage population to refrain from drinking. However, recent analyses of the lyrical context of current alcohol mentions in popular music demonstrate an alarmingly common positive perception of at-risk drinking behaviors, with frequent directionality toward America's youth and those under the current legal drinking age. ${ }^{24-26}$ Little published data support a stance that lyrical reference to drugs and alcohol can influence public consumption; however, studies have demonstrated that adolescents are more likely to consume alcohol when exposed to alcohol-related musical advertising. ${ }^{26-33}$ Music has persuasive capabilities, ${ }^{26-28}$ and Top 40's music harbors a potential to influence a vast audience, including young adults and adolescents. Although further studies may be warranted to evaluate this music's persuasive ability in regard to opioids or other drugs, understanding the commonality of drug and alcohol reference in popular music may stand to initiate larger and more in-depth analyses or to facilitate an open dialect between care providers and patients on health events related to drugs and alcohol.
To the best of our knowledge, our study brings to light previously undescribed trends in popular musical reference to opioids, among other drugs of interest. But, although the strengths of our study include its comprehensiveness and its detailed inquiry, we would be remiss not to mention a few limitations present in the current analysis. First, and probably of most importance, our study does not aim to contextualize any of the references identified. It is possible that the discovered lyrical increase has been a promotional plea for listeners to be wary of the dangerous effects of recreational drug use; although our study aimed to identify trends in lyrical commonality, a detailed contextual analysis was not undertaken. Although, with an identified dramatic uptrend in lyrical mention to these drugs of interest, further detailed inquiry may be justified to contextualize the references and lyrical messages delivered to Top 40's listeners. Second, our query used computer programming to identify drug-related keywords of interest in each song (Appendix A). Although we compiled a detailed list of searchable phrases and words to identify drugs of interest, it is possible and likely that our list is not all-inclusive. Furthermore, creatively penned emphasis in our lyrical sample may have caused rare cases of unidentified reference. Yet, with a clearly delineated Appendix of terminology, our hope is that readers can comfortably draw conclusions on the richness of our statistical review. Finally, readers must understand that the correlative analysis undertaken by our study makes no aim to imply causality. The opioid use disorder epidemic in the United States has numerous contributing factors, and this review evaluates only a small niche of social context relevant to the ongoing problem.

\section{Conclusion}

There is increasing prevalence in the lyrical mention of opioids, among other drugs, in Top 40's music. This increase has coincided with an increased occurrence of opioid use disorder in the United States. Nearly $50 \%$ of today's Top 40's songs reference drugs or alcohol. Further inquiry may be warranted to evaluate the societal impact and persuasive abilities of popular culture, including Top 40's music, on American drug and alcohol use. 
To see this article online, please go to: http://jabfm.org/content/ 31/5/761.full.

\section{References}

1. Martins SS, Sarvet A, Santaella-Tenorio J, Saha T, Grant BF, Hasin DS. Changes in US lifetime heroin use and heroin use disorder: prevalence from the 2001-2002 to 2012-2013 National Epidemiologic Survey on Alcohol and Related Conditions. JAMA Psychiatry 2017;74:445-455.

2. Jordan AE, Blackburn NA, Des Jarlais DC, Hagan H. Past-year prevalence of prescription opioid misuse among those 11 to 30years of age in the United States: a systematic review and meta-analysis. J Subst Abuse Treat 2017;77:31-37.

3. National Center for Health Statistics. Wide-ranging online data for epidemiologic research (WONDER). Available from: http://wonder.cdc.gov. Published 2016. Accessed July 11, 2017.

4. Substance Abuse and Mental Health Services Administration. 2015 National Survey on Drug Use and Health (NSDUH). Table 2.41B-Alcohol Use in Lifetime, Past Year, and Past Month among Persons Aged 12 or Older, by Demographic Characteristics: Percentages, 2014 and 2015. Rockville, MD: Center for Behavioral Health Statistics and Quality; 2016.

5. U.S. Department of Health and Human Services. The health consequences of smoking-50 years of progress: a report of the Surgeon General. Atlanta, GA: U.S. Department of Health and Human Services, Centers for Disease Control and Prevention, National Center for Chronic Disease Prevention and Health Promotion, Office on Smoking and Health; 2014.

6. Centers for Disease Control and Prevention. Current cigarette smoking among adults-United States, 2005-2015. MMWR 2016;65:1205-11.

7. Dunn, M. What is the relationship between art and society? Availablefrom:http://www.theoryofknowledge. net/areas-of-knowledge/the-arts/what-is-the-relation ship-between-art-and-society/. Published May 2013. Accessed July 13, 2017.

8. Centers for Disease Control and Prevention. Prescription opioid overdose data. Available from: https://www. cdc.gov/drugoverdose/data/overdose.html. Published 2017. Accessed July 13, 2017.

9. Centers for Disease Control and Prevention. Increases in fentanyl drug confiscations and fentanylrelated overdose fatalities; 2015. Available from: https://www.cdc.gov/drugoverdose/opioids/fentanyl. html. Accessed November 2017.

10. Maryland Department of Health. Drug and Alcohol Related Intoxication Deaths in Maryland; 2014. Available from: https://bha.health.maryland.gov/ OVERDOSE_PREVENTION/Pages/Data-andReports.aspx. Accessed November 2017.

11. Drug Enforcement Administration. DEA issues nationwide alert on fentanyl as threat to health and public safety; 2015. Available from: https://www.dea. gov/divisions/hq/2015/hq031815.shtml. Accessed November 2017.

12. Centers for Disease Control and Prevention. Increases in fentanyl drug confiscations and fentanylrelated overdose fatalities; 2015. Available from: https://emergency.cdc.gov/han/han00384.asp. Accessed November 2017.

13. Centers for Disease Control and Prevention. CDC guideline for prescribing opioids for chronic pain; 2016. Available from: https://www.cdc.gov/drugoverdose/ prescribing/guideline.html. Accessed November 2017.

14. Gallup, Inc. Support for legal marijuana use up to $60 \%$ in U.S. Available from: www.Gallup.com/poll/196550/ support-legal-marijuana.aspx. Published 2016. Accessed July 15, 2017.

15. Pew Research Center. Majority now supports legalizing marijuana. Available from: pewresearch.org. Published 2016. Accessed July 11, 2017.

16. U.S. Department of Health, Education, and Welfare. Smoking and health: report of the advisory committee to the Surgeon General of the Public Health Service; 1964. Available from: https://profiles. nlm.nih.gov/ps/access/nnbbmq.pdf. Accessed November 2017.

17. U.S. Department of Health and Human Services. The health consequences of smoking - 50 years of progress; 2014. Available from: https://www.surgeongeneral. gov/library/reports/50-years-of-progress/index.html. Accessed November 2017.

18. Bektas M, Ozturk C, Karatas H, Bektas I. Effects of student perceptions of social skills on their perception of smoking. Asian Pac J Cancer Prev 2014;15: 5937-40.

19. Zhang Y, Liu Y, Wang J, Jia C. Mediation of smoking consumption on the association of perception of smoking risks with successful spontaneous smoking cessation. Int J Behav Med 2014;21:677-81.

20. Alzyoud S, Kheirallah KA, Weglicki LS, Ward KD, Al-Khawaldeh A, Shotar A. Tobacco smoking status and perception of health among a sample of Jordanian students. Int J Environ Res Public Health 2014; 11:7022-35.

21. Leidner A, Shaw W, Yen S. An historical perspective on health-risk awareness and unhealthy behaviour: cigarette smoking in the United States 1949-1981. Health Expec; 2015;18:2720-30.

22. Cummings K, Proctor R. The changing public image of smoking in the United States: 1964-2014. Cancer Epidemiol Biomarkers Prev 2014;23:32-6.

23. Hedden S, Kennet J, Lipari R, Medley G, Tice P. Behavioral health trends in the United States: results from the 2014 National Survey on Drug Use and Health. U.S. Rockville, MD: Department of Health and Human Services; 2015.

24. Primack BA, Dalton MA, Carroll MV, Agarwal AA, Fine MJ. Content analysis of tobacco, alcohol, and 
other drugs in popular music. Arch Pediatr Adolesc Med 2008;162:169-175.

25. Siegel M, Johnson RM, Tyagi K, et al. Alcohol brand references in U.S. popular music, 2009-2011. Subst Use Misuse 2013;48.

26. Christenson P, Roberts DF, Bjork N. Booze, drugs, and pop music: trends in substance portrayals in the Billboard Top 100 - 1968-2008. Subst Use Misuse 2012;47:121-129.

27. Alpert J, Alpert M. Music influences on mood and purchase intentions. Psychol Mark 1990;7:109-33.

28. Niven K. Can music with prosocial lyrics heal the working world? A field intervention in a call center. J Appl Soc Psychol 2015;45:132-8.

29. Federal Trade Commission. Self-regulation in the alcohol industry: a review of industry efforts to avoid promoting alcohol to underage consumers. Available from: https://www.ftc.gov/reports/selfregulation-alcohol-industry-federal-trade-commissionreport-congress. Published 1999. Accessed 7/18/2017.

30. Snyder L, Milici F, Slater M, Sun H, Strizhakova Y, Effects of alcohol advertising exposure on drinking among youth. Arch Pediatr Adolesc Med 2006; 160: $18-24$.

31. Stacy A, Zogg J, Unger J, Dent C. Exposure to televised alcohol ads and subsequent adolescent alcohol use. Am J Health Behav 2004;6:498-509.

32. Ellickson P, Collins R, Hambarsoomians K, McCaffrey D. Does alcohol advertising promote adolescent drinking? Results from a longitudinal assessment. Addiction 2005;100:235-46.
33. Chen M, Grube J, Bersamin M, Walters E, Keefe D. Alcohol advertising: what makes it attractive to youth? J Health Commun 2005;10:202-21.

\section{Appendix A}

Marijuana Keywords: pot (reviewed), herb, chronic, cannabis, ganja, smoke (reviewed), green (reviewed), weed, blunt, marij, mary jane, vape, joint, brocc, kush, high (reviewed), grass (reviewed), dope (reviewed), spliff (reviewed), light up (reviewed)

Alcohol Keywords: drink (reviewed), henn (reviewed), drunk (reviewed), beer, liqu, Budweiser, crunk, booze, alcohol, dom per, rose (reviewed), rye (reviewed), bourbon, whisky, champagne, vodka, tequila, patron, my cup (reviewed), wine (reviewed), the red (reviewed), the white (reviewed), shot (reviewed)

Tobacco Keywords: cigarette, cigar, pipe (reviewed), marlboro, camels, cig, chaw, snuff, dip (reviewed), spitter, tobacco, smoke (reviewed), puff (reviewed), inhale (inhale), blow (reviewed), light up (reviewed)

Oploid Keywords: syrup (reviewed), dope (reviewed), heroin, oxy (reviewed), vicod, perc, phine, pills (reviewed), medicine (reviewed), meds (reviewed), sizzur, codeine, op??, purple dr, opiate, remy (reviewed), fent (reviewed) 